\title{
Amministrazione di sostegno, volontà del beneficiario e rifiuto delle cure
}

\author{
Paola Delbon ${ }^{1}$, Adelaide Conti ${ }^{1}$ \\ 1 Centro di Studio e di Ricerca di Bioetica dell'Università degli Studi di Brescia (in coll. con l'IRCCS Centro San Giovanni di Dio Fatebenefratelli). \\ Cattedra di Medicina Legale
}

\begin{abstract}
Many recent judgments, authorize the so called "amministratore di sostegno" (introduced in our legal system by Law n. 6 of January $9^{\text {th }}, 2004$ ) to express informed consent to medical treatments in the interest of the beneficiary/ patient - when he/ she is not able to make a reasonable assessment of his/ her interests, and then to express a valid consent to medical treatments - and even to refuse medical treatments, according to the wishes of the patients, on the ground of his/ her opinion, expressed in an advance care directive or resulting from his/ her behaviour, cultural background, ethical and religious convictions, etc.

The article examines some judgments of the Italian Court that can better explain the importance and the meaning of this figure.
\end{abstract}

Keywords: amministratore di sostegno, support administration procedure, incompetent patient, advance care directive, refusal of care

Amministratore di sostegno, patient's wishes, refusal of care Pratica Medica \& Aspetti Legali 2009; 3(1): 27-33

\section{INTRODUZIONE}

Le più recenti pronunce giurisprudenziali paiono univocamente orientate all'affermazione della centralità della volontà del paziente nel processo decisionale relativo ai trattamenti sanitari da attuarsi nei suoi confronti, in particolare in relazione all'ipotesi in cui l'interessato non sia in grado di manifestare personalmente la propria volontà ai curanti.

L'ipotesi di sopravvenuta incapacità del soggetto - in conseguenza di un evento non previsto o dell'evoluzione prevedibile della patologia da cui è affetto - genera infatti l'insorgenza della necessità di una riflessione atta a individuare quali strumenti contemplati dall'ordinamento giuridico possano rivestire una funzione di garanzia dell'attuazione della volontà della persona anche nel momento in cui quest'ultima non sia nelle con- dizioni di farla valere personalmente. In tale prospettiva, la giurisprudenza sembra aderire a una configurazione dell'istituto dell'amministrazione di sostegno, oltre che quale strumento di supporto nell'assistenza e nella cura del beneficiario, anche quale possibile strumento di affermazione dell'autodeterminazione dello stesso, anche in relazione a una futura - più o meno prossima nel tempo - (ed eventuale) situazione di incapacità dell'interessato.

\section{ANALISI DELLE PRONUNCE GIURISPRUDENZIALI}

Significativo, in tal senso, un pronunciamento del Giudice tutelare del Tribunale di Modena (Giudi- 
ce tutelare del Tribunale di Modena, decreto 13 maggio 2008, consultabile online su: http://www. personaedanno.it) che ha riconosciuto la possibilità di attribuire all'amministratore di sostegno la responsabilità dell'attuazione della volontà del beneficiario anche laddove volta al rifiuto di una pratica invasiva salvavita.

Nel caso di specie, il Tribunale di Modena si è pronunciato rispetto a una richiesta di amministratore di sostegno per una donna affetta da sclerosi laterale amiotrofica con grave insufficienza respiratoria in ventilazione meccanica non invasiva continua: la richiesta era sostenuta da una relazione neurologica e da una relazione psichiatrica che documentavano la capacità di intendere $\mathrm{e}$ di volere e la piena consapevolezza della donna, non affetta da alcun deficit cognitivo, nonché la sua ferma volontà contraria al possibile e a breve prevedibile ricorso a ventilazione invasiva, e da una dichiarazione del coniuge della donna in cui lo stesso affermava di accogliere la volontà della moglie chiedendo "che non venga effettuato l'intervento di tracheotomia, in caso di necessità» e ribadendo che tale volontà era stata espressa dalla donna in presenza dello stesso e dei figli.

L'esigenza della nomina di un amministratore di sostegno nel caso di specie è stata originata dalla considerazione che «le segnalate disabilità determinerebbero, per la persona, il serio rischio di una totale sua impossibilità di far valere la propria volontà nei termini sopra riferiti qualora, verificandosi una crisi respiratoria non superabile con i mezzi di cura in atto ed entrando essa in stato confusionale per la mancanza di ossigeno, i sanitari procedessero ad una ventilazione forzata invasiva con tracheotomia; di qui la richiesta di un indispensabile sostegno per sostituirla, una volta sopravvenuta la sua incapacità, nell'atto di diniego del consenso alla specifica pratica medica».

Il Giudice, interrogati il coniuge e i figli della donna, nonché visitata l'interessata, e appurata nella stessa, "senza tentennamenti, con piena lucidità e con coerenza delle risposte alle domande, la volontà precisa di non intendere di essere sottoposta alla pratica invasiva [...] contestualmente manifestando una coraggiosa coscienza delle conseguenze probabilmente infauste della propria scelta», emanava il decreto di nomina dell'amministratore di sostegno, autorizzandolo a compiere, in nome e per conto della beneficiaria:

- «negazione di consenso ai sanitari coinvolti a praticare ventilazione forzata e tracheotomia all'atto in cui, senza che sia manifestata contraria volontà della persona, l'evolversi della malattia imponesse, la specifica terapia salvifica;

- richiesta ai sanitari di apprestare, con la maggiore tempestività e anticipazioni con- sentite, le cure palliative più efficaci al fine di annullare ogni sofferenza alla persona».

Alcuni Autori [1], commentando il caso in esame, hanno evidenziato come "la perdita di coscienza non può tuttavia determinare anche la perdita di diritti fondamentali. Ci sono gli strumenti per evitare quella che altrimenti sarebbe una inammissibile violazione del principio di eguaglianza, per far valere l'autodeterminazione oltre la soglia della perdita di coscienza».

Il riconoscimento dell'amministrazione di sostegno quale strumento per far valere la volontà previamente manifestata dal soggetto emergeva già da alcuni pronunciamenti precedenti.

Il Giudice tutelare del Tribunale di Tivoli - sez. Palestrina, con decreto del 10 ottobre 2007 (http:// www.personaedanno.it) autorizzava l'amministratore di sostegno, nominato per un paziente Testimone di Geova in coma farmacologico e in prognosi strettamente riservata, a seguito di un incidente automobilistico, e che in precedenza aveva espresso il proprio incondizionato rifiuto alla terapia emotrasfusionale, «a manifestare ai sanitari la volontà a suo tempo espressa dal beneficiario in merito ad atti trasfusionali di sangue ed emoderivati ed a prestare il consenso informato ai trattamenti terapeutici necessari per la cura della salute fisica e psichica del beneficiario».

Il Giudice tutelare del Tribunale di Bologna - sez. di Imola, con decreto 4 giugno 2008 (http://www. personaedanno.it), autorizzava l'amministratore di sostegno di un paziente Testimone di Geova "a compiere in nome e per conto del beneficiario la negazione del consenso alle emotrasfusioni nel momento in cui, senza che sia stata manifestata contraria volontà da parte del (...), i sanitari ritenessero necessario praticarle ed il (...) fosse nell'impossibilità di esprimere le sue volontà al riguardo».

Il caso di specie riguardava un paziente "nell'imminenza di subire un intervento chirurgico al cuore che lo renderà, nel tempo in cui perdurerà l'intervento e durante l'anestesia, totalmente impossibilitato ad attendere ai propri interessi».

Il soggetto aveva dichiarato la propria volontà contraria all'effettuazione di trasfusioni, confermando quanto già trasmesso nelle direttive anticipate sottoscritte pochi giorni prima e aveva dunque presentato ricorso per ottenere la nomina di un amministratore di sostegno in proprio favore, alla luce del fatto che l'intervento imminente avrebbe potuto determinare «il rischio per lui di una sua totale impossibilità di fare valere la propria volontà nei termini sopra riferiti qualora i sanitari si trovassero nella necessità durante l'intervento, di praticare emotrasfusioni, da cui la necessità di un indispensabile sostegno per sostituirlo nell'atto di diniego del consenso alle trasfusioni, una volta sopravvenuta la sua incapacità a farlo». 


\section{NORMATIVA DI RIFERIMENTO}

L'istituto dell'amministrazione di sostegno è stato introdotto nel nostro ordinamento giuridico con Legge 9 gennaio 2004, n. 6 ("Introduzione nel libro primo, titolo XII, del codice civile del capo I, relativo all'istituzione dell'amministrazione di sostegno e modifica degli articoli 388, 414, 417, $418,424,426,427$ e 429 del codice civile in materia di interdizione e di inabilitazione, nonché relative norme di attuazione, di coordinamento e finali”), con l'intento di «tutelare, con la minor limitazione possibile della capacità di agire, le persone prive in tutto o in parte di autonomia nell'espletamento delle funzioni della vita quotidiana, mediante interventi di sostegno temporaneo o permanente» (art. 1).

Lart. 404 ("Amministrazione di sostegno"), introdotto nel Titolo XII del libro primo del codice civile, prevede che "la persona che, per effetto di una infermità ovvero di una menomazione fisica o psichica, si trova nella impossibilità, anche parziale o temporanea, di provvedere ai propri interessi, può essere assistita da un amministratore di sostegno, nominato dal giudice tutelare del luogo in cui questa ha la residenza o il domicilio».

Il nuovo istituto intende quindi predisporre uno strumento di sostegno per il soggetto non autonomo "con l'obiettivo non già di un asettico accertamento, di incapacità di agire collegato alla logica ghettizzante dell'infermità di mente $[. .$.$] con il$ corollario della mera sostituzione della persona in ogni atto giuridicamente rilevante; ma di realizzare uno strumento idoneo a sopperire per quanto possibile a tutte le carenze (impossibilità di provvedere ai propri interessi) della persona per qualsiasi ragione (per ogni infermità o menomazione) non autonoma [...], costruendo per lei [...] un progetto di sostegno più o meno limitato in dipendenza delle richieste e delle esigenze» [2].

La nuova disciplina pare peraltro riconoscere uno spazio significativo alla cura dell'incapace laddove, all'art. 405 cod. civ. ("Decreto di nomina dell'amministratore di sostegno. Durata dell'incarico e relativa pubblicità"), prevede che "qualora ne sussista la necessità, il giudice tutelare adotta anche d'ufficio i provvedimenti urgenti per la cura della persona interessata e per la conservazione e l'amministrazione del suo patrimonio», e all'art. 408 cod. civ. ("Scelta dell'amministratore di sostegno") secondo il quale «la scelta dell'amministratore di sostegno avviene con esclusivo riguardo alla cura ed agli interessi della persona del beneficiario».

La Legge n. 6/ 2004 non contiene peraltro un'esplicita disposizione riguardo alla possibile attribuzione all'amministratore di sostegno - se necessario nel caso di specie - della legittimazione ad esprimere il consenso ai trattamenti sanitari nell'interesse del beneficiario.
Nel caso in cui «la volontà del beneficiario non sia condizionata o viziata da una patologia o da una menomazione che gli impediscano una corretta rappresentazione dell'intervento terapeutico e delle conseguenze della sua realizzazione o omissione, in linea di principio non si potrà intervenire contro la sua libera volontà» [3]; d'altra parte la giurisprudenza prevalente riconosce la possibile attribuzione all'amministratore di sostegno della legittimazione ad esprimere il consenso ai trattamenti sanitari nell'interesse del beneficiario laddove tale legittimazione configuri uno strumento di cura ovvero di tutela della salute di un paziente non in grado di determinarsi consapevolmente in relazione alla stessa.

Il Giudice tutelare del Tribunale di Modena in data 28 giugno 2004 (Giudice tutelare del Tribunale di Modena, sez. civ., decreto 28 giugno 2004 [4]) emetteva decreto di nomina di amministratore di sostegno per un paziente psichiatrico, ricoverato presso un reparto di psichiatria e salute mentale, a fronte delle deliranti e confuse motivazioni con cui l'interessato, infortunatosi e in condizioni tali - in vista del rischio di prevedibili menomazioni future - da richiedere un intervento chirurgico, ribadiva il proprio dissenso ai trattamenti.

Il Giudice attribuiva all'amministratore di sostegno l'incarico di assumere ogni iniziativa utile affinché il paziente si sottoponesse al citato intervento, ovvero riconosceva all'amministratore una funzione di assistenza necessaria al beneficiario nell'attività di disposizione del proprio stato di salute.

Successivamente, il Giudice tutelare, a fronte della richiesta dell'amministratore di sostegno, riconoscendo il carattere di urgenza dell'intervento nell'interesse del beneficiario e al contempo rilevando il costante dissenso espresso da quest'ultimo, affetto da «un'alterazione grave del rapporto col reale con menomazioni così sensibili di pensiero, volontà e condotta da renderlo privo di consapevolezza critica della malattia e delle conseguenze che gliene possono derivare», autorizzava l'amministratore di sostegno stesso ad esprimere il consenso al trattamento, in nome e per conto del beneficiario.

Il Giudice sottolineava come la garanzia della "cura della persona" comporti «il coinvolgimento anche di quel diritto - dovere di esprimere il cosiddetto consenso informato che, se escluso, in ipotesi, qualora l'interessato non sia in grado di determinarsi (tale l'interdetto) comporterebbe ineludibile lesione della legislativamente consacrata funzione della "cura della persona"».

Nel caso di specie, dunque, il necessario riconoscimento dell'incapacità di determinarsi consapevolmente del paziente psichiatrico e al contempo la riconosciuta esigenza di garantire la "cura della persona" dello stesso, si traduceva nella legittimazione dell'amministratore di sostegno ad esprimere il consenso ai trattamenti. 
Così, il Giudice tutelare del Tribunale di Roma, nel marzo 2004 (Giudice tutelare del Tribunale di Roma, decreto 19 marzo 2004 [5]) ha stabilito che «può essere designato amministratore di sostegno ad una persona inabilitata ad esprimere il consenso al trattamento medico, attribuendogli il potere di esprimere tale consenso in suo nome e vece». Nel caso di specie il Giudice, alla luce della grave situazione in cui versava la paziente, che non pareva in condizioni di esprimere una volontà consapevole riguardo agli interventi medici anche chirurgici da attuarsi, e alla luce della "assoluta urgenza di provvedere alla cura dell'interessata», disponeva la nomina dell'amministratore di sostegno con l'incarico di dare «in nome e per conto della beneficiaria tutti i consensi che si rendessero necessari alla cura della stessa».

Del resto, l'art. 37 (“Consenso del legale rappresentante") del Codice di deontologia medica, approvato nell'ultima versione dalla Federazione Nazionale degli Ordini dei Medici Chirurghi e degli Odontoiatri in data 16 dicembre 2006, ed entrato in vigore in data 1 marzo 2007, dopo aver affermato che in caso di minore o di interdetto il consenso ai trattamenti sanitari è espresso dal rappresentante legale, precisa che «il medico, nel caso in cui sia stato nominato dal giudice tutelare un amministrazione di sostegno deve debitamente informarlo e tenere nel massimo conto le sue istanze».

Tale interpretazione trova conferma anche nell'art. 6 ("Protezione delle persone che non hanno la capacità di dare consenso") comma 3 della "Convenzione per la protezione dei Diritti dell'Uomo e della dignità dell'essere umano nei confronti delle applicazioni della biologia e della medicina: Convenzione sui Diritti dell'Uomo e la biomedicina", aperta alla firma degli Stati membri del Consiglio d'Europa a Oviedo in data 4 aprile 1997, e di cui l'Italia ha autorizzato la ratifica con Legge 28 marzo 2001, n. 145: tale norma stabilisce infatti che «allorquando, secondo la legge, un maggiorenne, a causa di un handicap mentale, di una malattia o per un motivo similare, non ha la capacità di dare consenso ad un intervento, questo non può essere effettuato senza l'autorizzazione del suo rappresentante, di un'autorità o di una persona o di un organo designato dalla legge. La persona interessata deve nei limiti del possibile essere associata alla procedura di autorizzazione».

Il richiamo a tale norma è contenuto anche nel decreto con cui il Giudice tutelare del Tribunale di Modena (Giudice tutelare del Tribunale di Modena, decreto 15 settembre 2004, http://www.personaedanno.it) autorizzava l'amministratore di sostegno di un paziente affetto da un disturbo delirante cronico nonché da una grave forma di diabete, di fronte all'assoluto dissenso del beneficiario a sottoporsi a qualsiasi terapia di cura della propria salute, e all'incapacità dello stesso di esprimere un valido consenso all'indispensabile sottoposi- zione a cure e terapie antidiabetiche, «ad esprimere in nome e per conto del beneficiario, il consenso informato ai trattamenti terapeutici proposti dai medici, se ed in quanto richiesti e/ o necessari, ponendo in essere ogni iniziativa conseguente». Così, il Giudice tutelare del Tribunale di Reggio Emilia, con decreto 13 settembre 2006 (http:// www.personaedanno.it) nominava l'amministratore di sostegno per un soggetto affetto da sclerosi laterale amiotrofica che, "mostrandosi adeguatamente consapevole delle proprie gravissime condizioni di salute», comportanti «un'evidente limitazione della capacità relazionale e dell'autonomia funzionale con riguardo allo svolgimento delle attività quotidiane di tipo strumentale, ivi incluse quelle connesse alla cura della propria persona ed alla gestione del proprio patrimonio» confermava «la necessità di essere opportunamente assistito nella cura dei propri interessi, sia con riferimento alle necessità terapeutiche che alle attività attinenti alla cura dei propri interessi patrimoniali». In particolare, il Giudice tutelare attribuiva all'amministratore di sostegno «in generale, il controllo e la vigilanza in relazione ad interventi e/ o prescrizioni e/ o trattamenti assistenziali, terapeutici e riabilitativi (ivi incluso, ove occorra e risulti giustificato da effettive esigenze rappresentate dai sanitari, la prestazione del consenso informato, cui il beneficiario dovesse in ipotesi trovarsi in condizioni tali da non essere in grado di provvedere in modo autonomo e consapevole)».

Il Giudice tutelare del Tribunale di Trieste, con decreto 2 luglio 2008 (http://www.personaedanno. it), nominava l'amministratore di sostegno per un paziente in stato di coma vigile da anossia cerebrale, conferendogli la legittimazione ad «assumere in nome e per conto del beneficiario decisioni sia in ordine alla gestione del suo patrimonio [...] sia in ordine alle modalità di assistenza e alla cura esprimendo anche il consenso informato per ogni trattamento sanitario».

In particolar modo, nel decreto di nomina l'amministratore di sostegno era esplicitamente autorizzato ad «acquisire referti e cartelle cliniche nonché ogni altra informazione di natura sanitaria e prestare il consenso informato per i trattamenti sanitari inerenti il Beneficiario».

La configurazione dell'amministrazione di sostegno, non solo quale strumento di cura della persona del beneficiario, ma anche quale mezzo di valorizzazione dell'autonomia decisionale dello stesso, trova conferma in quanto previsto dalla normativa in materia.

Anzitutto l'art. 410 cod. civ. ("Doveri dell'amministratore di sostegno") stabilisce che «nello svolgimento dei suoi compiti l'amministratore di sostegno deve tener conto dei bisogni e delle aspirazioni del beneficiario.

L'amministratore di sostegno deve tempestivamente informare il beneficiario circa gli atti da 
compiere, nonché il giudice tutelare in caso di dissenso con il beneficiario stesso. In caso di contrasto, di scelte o di atti dannosi ovvero di negligenza nel perseguire l'interesse o nel soddisfare i bisogni o le richieste del beneficiario, questi, il pubblico ministero o gli altri soggetti di cui all'articolo 406 possono ricorrere al giudice tutelare, che adotta con decreto motivato gli opportuni provvedimenti».

Lart. 408 cod. civ. ("Scelta dell'amministratore di sostegno") prevede che "la scelta dell'amministratore di sostegno avviene con esclusivo riguardo alla cura ed agli interessi della persona del beneficiario. L'amministratore di sostegno può essere designato dallo stesso interessato, in previsione della propria eventuale futura incapacità, mediante atto pubblico o scrittura privata autenticata».

D'altra parte l'art. 406 cod. civ. ("Soggetti”) afferma che «il ricorso per l'istituzione dell'amministrazione di sostegno può essere proposto dallo stesso soggetto beneficiario».

La previsione della possibile designazione dell'amministratore di sostegno direttamente da parte dell'interessato e il riconoscimento della stessa quale criterio preferenziale nella scelta del Giudice consente la proiezione della volontà attuale del soggetto nel tempo futuro della non coscienza: «la scelta di una persona di fiducia offre maggiori garanzie che le decisioni, specie quelle di natura esistenziale, vengano prese nel rispetto della personalità del beneficiario. La legge non lo dice espressamente, ma appare coerente con il suo impianto complessivo ritenere che a questa persona il malato possa dare anche direttive anticipate sulle decisioni che gli stanno più a cuore» [6].

Le norme citate sono state recentemente richiamate anche in una pronuncia del Giudice tutelare del Tribunale di Modena, che, con decreto 5 novembre 2008 (disponibile su: http://www.altalex.com), interveniva sul ricorso presentato da un soggetto per la nomina di amministratore di sostegno in favore di se stesso, sulla base di una previa scrittura privata autenticata in cui l'interessato designava la moglie come proprio amministratore di sostegno con l'incarico di garantire - nell'ipotesi di una futura eventuale situazione di incapacità del disponente - l'adempimento delle disposizioni di rifiuto dei trattamenti terapeutici e di consenso rispetto alle cure palliative, trasmesse dal soggetto nello stesso documento, e da attuarsi «in caso di malattia allo stadio terminale, malattia o lesione traumatica cerebrale, irreversibile e invalidante», ovvero una malattia che costringa il soggetto a "trattamenti permanenti con macchine o sistemi artificiali che impediscano una normale vita di relazione».

Il Giudice, in relazione al caso di specie e in particolare alla possibilità di nomina dell'amministratore di sostegno da parte dell'interessato in previsione della propria futura incapacità ai sensi dell'art. 408 cod. civ., rilevava come «la lettera della disposizione, la sua ratio, l'enunciazione, infine, nell'ambito di una disciplina tutta incentrata sulla tutela della persona e delle sue esigenze esistenziali, autorizzano e legittimano la constatazione che l'amministratore di sostegno è, nell'attualità, l'istituto appropriato per esprimere quelle disposizioni appropriate sui trattamenti sanitari per l'ipotesi di incapacità che vanno usualmente sotto il nome di testamento biologico».

Nel caso in esame dunque era riconosciuto al soggetto nominato amministratore di sostegno la possibilità di avvalersi, nel momento in cui si rendesse necessario, di una autorizzazione ottenuta in precedenza, a negare il consenso ai trattamenti sanitari: tale autorizzazione, secondo i Giudici, sarebbe da considerarsi doverosa ai sensi dell'art. 32 Cost. quale norma a garanzia del fatto che il naturale evento della morte si realizzi "con modalità coerenti all'autocoscienza della dignità personale quale costruita dall'individuo nel corso della vita attraverso le sue ricerche razionali e le sue esperienze emozionali».

Secondo i Giudici ciò consentirebbe anche di superare «l'impossibilità del mandatario di ottenere in tempo reale il decreto di nomina dell'amministratore»: del resto la stessa lettera dell'art. 404 c.c. sembra suggerire che «il Legislatore ha individuato l'attualità dello stato di incapacità del beneficiario come presupposto per la produzione degli effetti dello strumento protettivo ma non anche come requisito per la sua istituzione».

L'individuazione di un terzo soggetto quale garante della volontà precedentemente espressa dall'interessato è prospettata anche in una recente sentenza della Suprema Corte [7], nella quale, in relazione all'ipotesi del rifiuto di emotrasfusioni in caso di paziente Testimone di Geova che si trovi in stato di incoscienza, si afferma l'esigenza che in tal caso, «a manifestare il dissenso al trattamento trasfusionale, sia o lo stesso paziente che rechi con sé una articolata, puntuale, espressa dichiarazione dalla quale inequivocamente emerga la volontà di impedire la trasfusione anche in ipotesi di pericolo di vita, ovvero un diverso soggetto da lui stesso indicato quale rappresentante ad acta il quale, dimostrata l'esistenza del proprio potere rappresentativo in parte qua, confermi tale dissenso, all'esito della ricevuta informazione da parte dei sanitari».

La possibile individuazione di una persona di fiducia che assicuri l'attuazione della volontà espressa dall'interessato o addirittura assuma decisioni nell'interesse di quest'ultimo, è frequentemente contemplata anche nella produzione de iure condendo in materia di dichiarazioni anticipate di trattamento.

I progetti di legge presentati ed attualmente in corso di esame in commissione contemplano infatti la figura del fiduciario, indicandone i compiti 
tra i quali «agire nell'esclusivo e migliore interesse della persona che gli ha dato mandato»; "tenere conto scrupolosamente delle indicazioni sottoscritte dalla persona nella dichiarazione di trattamento anticipata»; «ricostruire il senso e il significato delle decisioni sottoscritte dal paziente»; «far conoscere queste volontà sia nel contesto sanitario sia in quello familiare per garantirne l'attuazione» (Disegno di legge n. 994, "Disposizioni in materia di dichiarazione anticipata di trattamento", presentato al Senato in data 4 agosto 2008), Così, l'art. 4 (Nomina del fiduciario) del Disegno di legge n. 136, «Disposizioni in materia di consenso informato e di dichiarazioni anticipate nei trattamenti sanitari», presentato al Senato in data 29 aprile 2008, stabilisce che "La dichiarazione anticipata $[. .$.$] può contenere l'indicazione di una per-$ sona di fiducia alla quale sono attribuite la titolarità, in caso di incapacità dell'interessato, dei diritti e delle facoltà che a questi competono ai sensi della presente legge nonché la tutela del rispetto da parte dei sanitari delle direttive espresse dallo stesso interessato» e che «il fiduciario agisce in conformità alle volontà del paziente».

Per quanto riguarda d'altra parte i criteri che lo stesso fiduciario dovrebbe adottare nel processo decisionale relativo ai trattamenti sanitari riguardanti il beneficiario, l'art. 5 (Miglior interesse) del Disegno di legge n. 800, "Direttive anticipate di fine vita", presentato al Senato in data 18 giugno 2008, prevede che «Colui che presta o rifiuta il consenso ai trattamenti [...], per conto di altri che versi in stato di incapacità, è tenuto ad agire nell'esclusivo e miglior interesse dell'incapace, tenendo conto della volontà espressa da quest'ultimo in precedenza, nonché dei valori e delle convinzioni da lui notoriamente espressi».

Anche nella Relazione che accompagna il Disegno di legge n. 10, "Disposizioni in materia di consenso informato e di dichiarazioni di volontà anticipate nei trattamenti sanitari al fine di evitare l'accanimento terapeutico, nonché in materia di cure palliative e di terapia del dolore", presentato al Senato in data 29 aprile 2008, si sottolinea che «In presenza di dichiarazioni anticipate di volontà è fondamentale tuttavia la presenza di un garante, definito "fiduciario" [...] che tuteli le indicazioni, ma che sappia, anche, sulla base di un solido e significativo rapporto personale, interpretare le volontà del paziente».

Indubbiamente la riflessione intorno alla possibilità di nomina di una persona di fiducia nelle dichiarazioni anticipate di volontà si inserisce nell'ambito della più ampia discussione relativa alla «operatività del meccanismo rappresentativo, nel rapporto che si instaura tra paziente e medico o struttura sanitaria, con particolare riferimento al consenso ad uno specifico trattamento» [8].

Le più recenti pronunce giurisprudenziali prese in esame rivelano nell'interpretazione - applicazio- ne delle norme dell'ordinamento giuridico la tendenza a riconoscere l'efficacia di tale meccanismo rappresentativo anche nell'ambito del processo decisionale relativo ai trattamenti sanitari, e anche in relazione all'espressione di un rifiuto di determinati trattamenti, purché ancorato al doveroso rispetto della volontà - espressa o ricostruita - dell'interessato.

Del resto, tale posizione evidenzia la necessaria considerazione che "nel processo clinico che riguarda adulti non più coscienti non sono coinvolte solamente considerazioni fattuali o tecniche di cui è depositario il medico [...] bensì anche, e spesso soprattutto, considerazioni valoriali un tempo anche queste affidate unicamente al medico e oggi rivendicate come gestibili dai soli soggetti coinvolti, quando possibile e nella misura in cui è possibile» [9]

Lo stesso Codice di deontologia medica stabilisce all'art. 38 (Autonomia del cittadino e direttive anticipate) che «il medico deve attenersi, nell'ambito dell'autonomia e indipendenza che caratterizza la professione, alla volontà liberamente espressa della persona di curarsi e deve agire nel rispetto della dignità, della libertà e autonomia della stessa» e che «il medico, se il paziente non è in grado di esprimere la propria volontà, deve tenere conto nelle proprie scelte di quanto precedentemente manifestato dallo stesso in modo certo e documentato».

I più recenti pronunciamenti giurisprudenziali tendono dunque a valorizzare l'autonomia e la centralità della soggettività dell'interessato nel quadro dell'ordinamento giuridico vigente: i Giudici (Trib. Modena, decreto 13 maggio 2008) evidenziano addirittura l'«assoluta superficialità di un intervento del legislatore volto a introdurre e disciplinare il c.d. testamentario biologico. Già esistono, infatti, il diritto sostanziale (artt. 2, 13 e 32 Cost.), lo strumento a mezzo del quale dare espressione alle proprie volontà (l'atto pubblico o la scrittura privata autenticata, art. 408 , comma 2 , c.c. cit.) e, infine, l'istituto processuale di cui avvalersi (l'amministrazione di sostegno, legge n. 6 del 2004)».

Il diritto all'autodeterminazione risulta così affermato nelle più recenti pronunce giurisprudenziali anche in relazione ai soggetti incapaci, sia per quanto riguarda il soggetto che abbia lasciato "specifiche disposizioni di volontà volte ad escludere trattamenti salvifici artificiali che lo mantengano in vita in stato vegetativo", sia anche in caso di paziente incapace «rispetto al quale il Giudice si formi il convincimento, sulla base di elementi probatori convincenti, che la complessiva personalità dell'individuo cosciente era nel senso di ritener lesiva della concezione stessa della sua dignità la permanenza e la protrazione di una vita vegetativa» (Trib. Modena, decreto 13 maggio 2008).

Anche la normativa relativa all'amministrazione di sostegno, nonché le modalità applicative di 
tale istituto, confermano l'orientamento in forza del quale «le decisioni di natura personale, anche quelle relative alla salute, debbono per quanto possibile essere il risultato di un'intesa tra beneficiario ed amministratore», e dunque in caso di incapacità di quest'ultimo «l'amministratore dovrà comunque conformare le proprie scelte al rispetto della personalità dell'interessato ed eventualmente tener conto delle volontà precedentemente espresse» [10].

\section{BIBLIOGRAFIA}

1. Ferrando G. Il diritto di rifiutare le cure e le direttive anticipate. Bioetica. Rivista Interdisciplinare 2008; 2: 336

2. Trentanovi S. Protezione delle persone prive di autonomia: rapporti tra amministrazione di sostegno e interdizione/ inabilitazione. Profili processuali e organizzativi. Rivista di Diritto delle Professioni Sanitarie 2004; 4: 143-4

3. Montanari Vergallo G. Il rapporto medico-paziente. Milano: Giuffrè, 2008; p. 158

4. Giudice tutelare del Tribunale di Modena, sez. civile, decreto del 28 giugno 2004. Diritto e Giustizia 2004; $30: 75$

5. Calò E. Amministrazione di sostegno. Legge 9 gennaio 2004, n. 6. Milano: Giuffrè, 2004; Appendice

6. Ferrando G. Il diritto di rifiutare le cure e le direttive anticipate. Bioetica. Rivista Interdisciplinare 2008; 2: 337-338

7. Corte di Cassazione, sentenza n. 23676 del 15 settembre 2008. Disponibile online su: http://www.personaedanno.it

8. Bailo R, Cecchi P, Direttive anticipate e diritto a rifiutare le cure: aspetti etici e giuridici, Rass. Dir. Civ., 1998; 3: 486

9. Canavacci L. La carta di autodeterminazione e il consenso informato. In: Dameno R (a cura di). Autodeterminarsi nonostante. Atti del Convegno "Verso il riconoscimento giuridico della carta di autodeterminazione. Un confronto europeo", 11-12 novembre. Milano: Guerini e Associati, 2002; p. 185

10. Ferrando G. Il diritto di rifiutare le cure e le direttive anticipate. Bioetica. Rivista Interdisciplinare 2008; 2: 337 